\title{
LCMS Measurement of Steroid Biomarkers Collected from Palmar Sweat.
}

2

3

24 Keywords: eccrine sweat, sweat biomarkers, non-invasive measurement, surface capture, $25 \mathrm{OIF} / \mathrm{OEF} / \mathrm{OND}$, precision medicine
J. Ray Runyon ${ }^{1,2 *}$ Jacob N. Hyde ${ }^{1,3}$, Christina Staroschak ${ }^{3,4}$, Bryan Kromenacker ${ }^{4,5}$, Robert C. Wilson ${ }^{5}$ Esther M. Sternberg ${ }^{1,4}$

${ }^{1}$ Andrew Weil Center for Integrative Medicine, University of Arizona, Tucson, Arizona, United States of America

${ }^{2}$ Department of Environmental Sciences, University of Arizona, Tucson, Arizona, United States of America

${ }^{3}$ Department of Family \& Community Medicine, College of Medicine, University of Arizona, Tucson, Arizona, United States of America

${ }^{4}$ College of Medicine, University of Arizona, Tucson, Arizona, United States of America

${ }^{5}$ Department of Psychology, College of Science, University of Arizona, Tucson, Arizona, United States of America

*Corresponding Author: J. Ray Runyon, PhD. jrayrunyon@arizona.edu 303-995-2624 
Abstract

27 Human eccrine sweat contains numerous biomarkers which can provide information on health, 28 performance, and aging. Non-invasive collection and measurement of biomarkers has become

29 especially important in recent times given viral outbreaks like SARS-CoV-2. In the current study

30 we describe a method of sweat collection from palmar surfaces in participants via surface capture

31 using glass beads and the resulting analysis of biomarkers from very low volumes of sweat using

32 liquid chromatography mass spectrometry with selected ion monitoring. Study participants

33 underwent a cognitive and physical stress task with easy and hard conditions with sweat being

34 collected after each task. Resulting analysis found a signal for 22 steroid biomarkers and we

35 report detailed information on selected biomarkers, given their applicability to timely real-world

36 exemplars, including cortisol, dehydroepiandrosterone, allopregnanolone, estrone, aldosterone,

37 and $20 \alpha / \beta$-dihydrocortisone. 


\subsection{Introduction}

50 Sweat is a biofluid rich in biomarkers that can provide information about human cognitive

51 performance, health, disease state(s), nutrition and environmental impacts. Steroids and other

52 immune biomarkers have been detected in eccrine sweat (1-12). Non-invasive measurement tools

53 of biomarkers are needed and have become especially salient given recent viral outbreaks like

54 SARS-CoV-2, where in-person blood collection is not feasible or unadvisable. The monitoring of

55 some biomarkers may help to monitor human performance in industrial and occupational

56 settings, may help to predict and track acquired disease processes like obesity, and may help to

57 determine how psychiatric sequalae are experienced and expressed.

59 Steroids of interest for the monitoring and enhancement of human performance and health are

60 many and include cortisol, dehydroepiandrosterone (DHEA), allopregnanolone, estrone and

61 aldosterone. Cortisol is secreted in response to stress, suppressing the hypothalamic-pituitary-

62 adrenocortical (HPA) axis and negatively effecting health and cognition (13). DHEA is

63 implicated in many physiological processes with impacts on disease resistance and immune

64 function (14). DHEA and cortisol are the most common products of the stress response from the

65 endocrine system, mediating short and long-term stress responses via the HPA-axis; DHEA can

66 be converted into dehydroepiandrosterone sulfate (DHEA-S) and has been shown to antagonize

67 the effects of cortisol (15). In humans, basal DHEA levels can be altered by exposure to

68 traumatic events like military combat and lower cortisol/DHEA ratios have been found in

69 patients with post-traumatic stress disorder (PTSD) (16). Positive correlations have also been

70 found between current PTSD symptoms and the ratio of DHEA to allopregnanolone (17).

71 Allopregnanolone is a metabolite of progesterone and plays a role in neuronal excitability at the 
72 synaptic and extrasynaptic $y$-aminobutyric acid $\left(\mathrm{GABA}_{\mathrm{A}}\right)$ receptor $(18)$; GABAergic

73 neurotransmission has been shown to be impaired in subjects with PTSD (19). Furthermore,

74 allopregnanolone is a key therapeutic target for research and development of neurodegenerative

75 and age-related diseases (20). Estrone is one of the three most common estrogens found in

76 humans. In men, estrone has been shown to increase as BMI rises (21) and may be a sensitive

77 marker of acquired Type-II diabetes risk (22). Aldosterone, through the Renin-Angiotensin-

78 Aldosterone System (RAAS) has health implications for the vascular, renal and cardiovascular

79 systems, which is of heightened interest due to the SARS-CoV-2 virus and resulting COVID-19

80 disease (23).

82 Steroid compounds of interest in human health and performance have been measured in plasma,

83 serum, urine, saliva, tissue and sweat $(4,11-12,23)$. Liquid chromatography/mass spectrometry

84 (LCMS) has been used to investigate the production and clearance of free cortisol (via

85 downstream inactive metabolites) in human eccrine sweat during heat and/or exercise induced

86 stress with sweat cortisol concentrations being similar to those measured in saliva $(4,12)$.

88 Analysis of target biomarkers in sweat has typically required a defined volume of sweat

89 necessary to apply benchtop assays for measurement or have utilized wearable devices for

90 collection via exercise stress $(5,12,25)$. Using pouches, wrist worn tubes (e.g. Macroduct), skin

91 surface scraping, vacuuming of sweat droplets, skin swabbing with absorbent materials, glass

92 rollers and collecting sweat from a whole body rinse have all been previously employed to

93 collect volumes of human eccrine sweat needed for analysis (26,27). 2-D molecular mapping of

94 latent finger prints using LCMS with electrospray ionization (LCMS-ESI) has been successful in 
95 identifying gender, age, ethnicity and disease markers of human subjects with over $80 \%$

96 accuracy showing that monitoring of biomarkers in very low volumes of secretions via surface

97 capture is possible(28).

98

99 In the current study, we explore the possibility of utilizing glass beads to collect very small

100 volumes of eccrine sweat via surface capture from the palms of the hands for targeted steroid

101 biomarker analysis in response to stress. Furthermore, we utilize a novel washing method to

102 extract the target biomarkers from the glass beads which simplifies sample preparation and clean

103 up in comparison to standard approaches such as solid phase extraction that is often used for

104 plasma and urine biofluid samples. Finally, the developed approach inclusive of sample

105 collection, sample preparation and LCMS methodology allows for robust analysis of non-

106 derivatized steroids thereby simplifying the analysis. Our discussion of results focuses

107 predominately on cortisol, DHEA, allopregnanolone, aldosterone and estrone given interest in

108 enhancement of human performance as mentioned earlier. We hypothesized that collection of

109 palmar sweat via surface capture would be a non-invasive, rapid, easily scalable method which

110 would yield sweat samples rich in molecular information correlating with physiological systems,

111 and provide a sensitive method for measuring biomarker changes on a time scale of minutes in

112 response to stress.

\section{$113 \quad 2.0$ Materials and Methods}

\section{$114 \quad 2.1$ Participants}

115 All participants gave informed consent before taking part in the experiment. The experiment was 116 approved by the University of Arizona Institutional Review Board. Participants were university 
117 students recruited through a program enabling them to receive educational credit for

118 participation. 5 participants took part in the study (1 male, 4 female; ages 18-22).

\section{$119 \quad 2.2$ Stress tasks and sample collection}

120 Study participants were asked to complete a series of cognitive and physical tasks on a computer

121 over the course of 45 minutes during which palmar sweat samples were collected concurrently

122 with real-time, high-resolution thermographic imaging of sweat pore activation from the left

123 lateral malleolus. Of note, this current manuscript focuses on the collection and characterization

124 of biomarkers collected from palmar sweat rather than thermographic imaging. A concurrent

125 report details the thermal imaging of sweat pore activation (29).

127 In this study, participants followed prompts on the screen from a program designed in

128 MATLAB. A neurocognitive task (N-back 1\&2 digits) was presented with two difficulty

129 conditions (easy/hard). Participants then engaged with a physical task (finger/keyboard tapping)

130 with two difficulty conditions (easy/hard) which were randomized within task for each

131 participant.

133 A batch of $4 \mathrm{~mm}$ glass beads made from borosilicate were baked at $475^{\circ} \mathrm{C}$ for 4 hours to remove 134 organic contaminates, allowed to return to room temperature, divided into 35 bead aliquots and 135 stored at room temperature in falcon tubes until use. Participants had these glass beads poured 136 from the tubes into their hands and then instructed to roll them between the palms of their hands 137 for 60 seconds. After collection of these samples, the beads were returned to the containers, and 138 then were stored at $4{ }^{\circ} \mathrm{C}$ until biomarker analysis. Sample collection was performed at the 
139 beginning of the study session, after completion of N-back cognitive tasks, and after completion 140 of physical tasks.

\section{$141 \quad 2.2$ LCMS-ESI-SIM of steroid biomarkers}

142 LCMS with electrospray ionization and selected ion monitoring (LCMS-ESI-SIM;

143 ThermoScientific TSQ Quantiva) was used to analyze the extract of steroid biomarkers from the

144 glass beads. Four $4 \mathrm{~mm}$ glass beads from each participant were washed with $50 \mu \mathrm{L}$ of $70 / 30$

145 LCMS water/ACN (1\% formic acid) in glass LCMS vials and then transferred to a second

146 LCMS vial with a clean glass insert. $2 \mu \mathrm{L}$ of glass bead extract was injected into the instrument

147 for analysis by LCMS-ESI-SIM. Chromatography of the extract was accomplished using a

148 Restek Raptor Biphenyl column (200 mm x $2.1 \mathrm{~mm} ; 2.7$ um bead) held at 30C and a water/ACN

149 ( $0.1 \%$ formic acid) binary solvent system. The solvent flow rate was set to $0.5 \mathrm{~mL} / \mathrm{min}$ with a

150 gradient of $\mathrm{ACN}$ from $10 \%-100 \%$ over 3 minutes followed by a column washing step for 1-

151 minute at $100 \% \mathrm{ACN}$ and a 3-minute column equilibrium step at $10 \% \mathrm{ACN}$ before the next

152 sample injection. Mass spectrometer settings used for detection of steroid biomarkers were as

153 follows: $3 \mathrm{kV}$ in positive ion mode, 0.4 resolution, $20 \mathrm{usec}$ dwell time, $300{ }^{\circ} \mathrm{C}$ vaporizer

154 temperature and ion transfer tube temperature, 50abs $\mathrm{N}_{2}$ sheath gas, $25 \mathrm{Arb} \mathrm{N}_{2}$ aux gas. Table 1

155 shows the steroid biomarkers tracked in the extract samples.

$157 \quad$ Table 1. Steroid Biomarker Surveyed in Palmar Sweat

\begin{tabular}{|l|l|l|}
\hline Steroid target biomarker & Mass $[\mathbf{m} / \mathbf{z}+\mathbf{1} ;+\mathbf{H}]$ & Steroid classification \\
\hline Estrone & 269 & \multirow{3}{*}{ Estrogen } \\
\hline Dihydroepiandrosterone (DHEA) & 271 & \multirow{2}{*}{ Androgen } \\
\hline Etiocholanolone & 273 & \\
\hline Androsterone & 273 & \\
\hline Androstenedione & 287 & \\
\cline { 1 - 2 } Testosterone & 289 & \\
\hline
\end{tabular}




\begin{tabular}{|c|c|c|}
\hline Dihydrotestosterone & 291 & \\
\hline Progesterone & 315 & \multirow{6}{*}{ Progestogen } \\
\hline 17a-hydroxypregnenolone & 315 & \\
\hline Pregnenolone & 317 & \\
\hline Pregnanolone & 319 & \\
\hline Allopregnanolone & 319 & \\
\hline 17a-hydroxyprogesterone & 331 & \\
\hline 11-deoxycorticosterone & 331 & \multirow{8}{*}{ Corticosteroids } \\
\hline 11-deoxycortisol & 347 & \\
\hline Corticosterone & 347 & \\
\hline Aldosterone & 359 & \\
\hline Cortisone & 361 & \\
\hline $20 \alpha / \beta$-dihydrocortisone $(20 \alpha / \beta$-DHCN) & 363 & \\
\hline Cortisol & 363 & \\
\hline $20 \alpha / \beta$-dihydrocortisol (20 $\alpha / \beta$-DHCL) & 365 & \\
\hline Cholesterol & 387 & \\
\hline
\end{tabular}

\section{$159 \quad 3.0$ Results}

160 The results shown below in Figure 1 summarize the different steroid molecules that were found

161 in palmar sweat utilizing an adapted LCMS methodology from (11). 

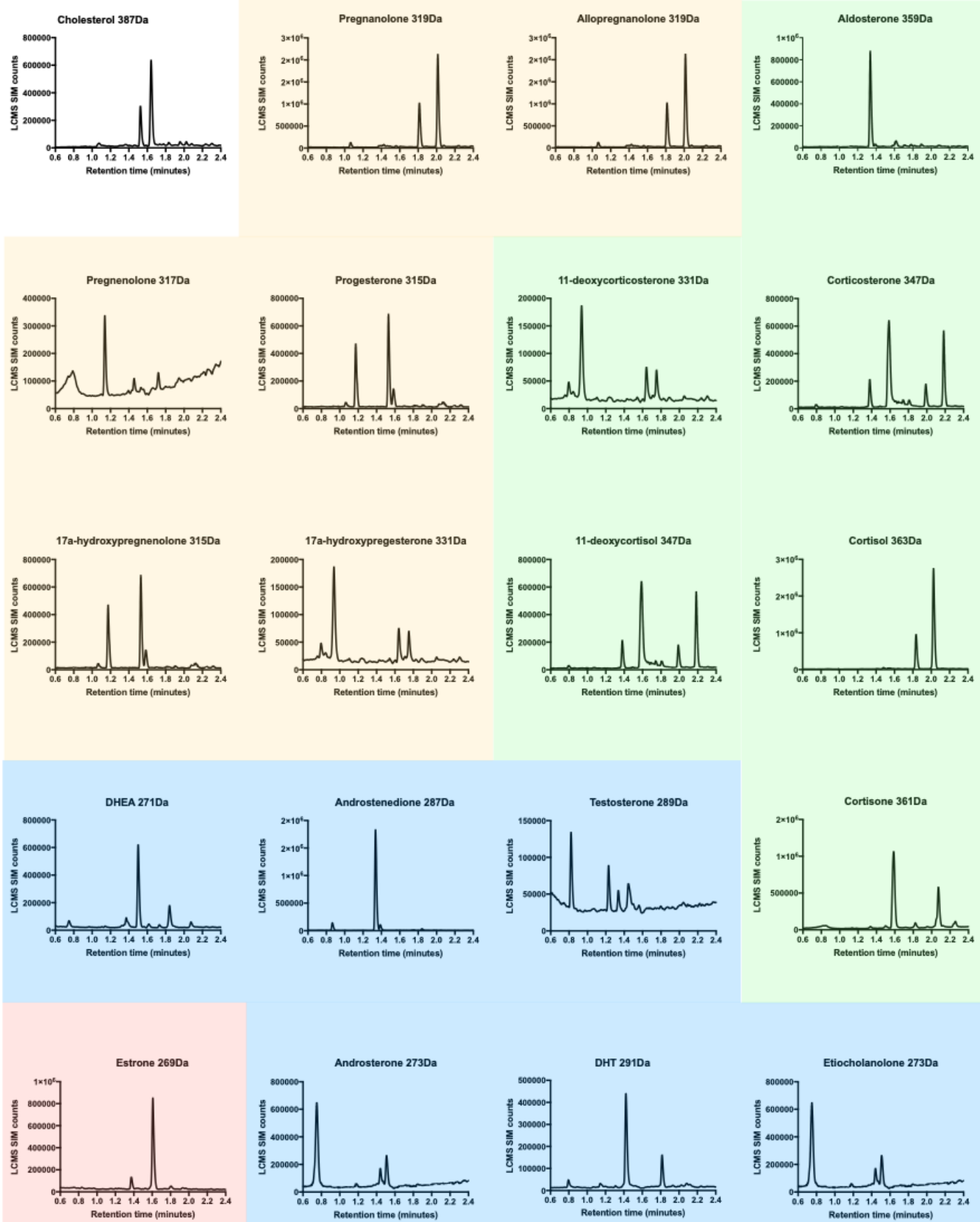

Figure 1. LCMS-ESI-SIM chromatograms for steroid biomarkers found in palmar sweat.

164 Progestogens (yellow), corticosteroids (green), androgens (blue), estrogens (pink), cholesterol 165 (white).

166 


\section{$167 \quad 3.1$ Stress induced changes in steroid biomarkers}

168 Healthy human volunteers were subjected to both cognitive and physical stress challenges on a

169 computer. Figures $2 \& 3$ present aggregated data for cortisol, $20 \alpha / \beta$-dihydrocortisone (a

170 metabolite of cortisol), DHEA, estrone, allopregnanolone, and aldosterone after completion of

171 each challenge. Figure 2 shows the biomarker response to a cognitive challenge as a percent

172 change in measured signal. Generally, a decrease in signal was observed for most biomarkers

173 across participants. Figure 3 shows the biomarker response to a physical challenge as a percent

174 change in measured signal as well. Generally, an increase was observed for most biomarkers

175 across participants. For both types of challenges, significant biomarker responses were observed

176 within minutes of the challenge.

177

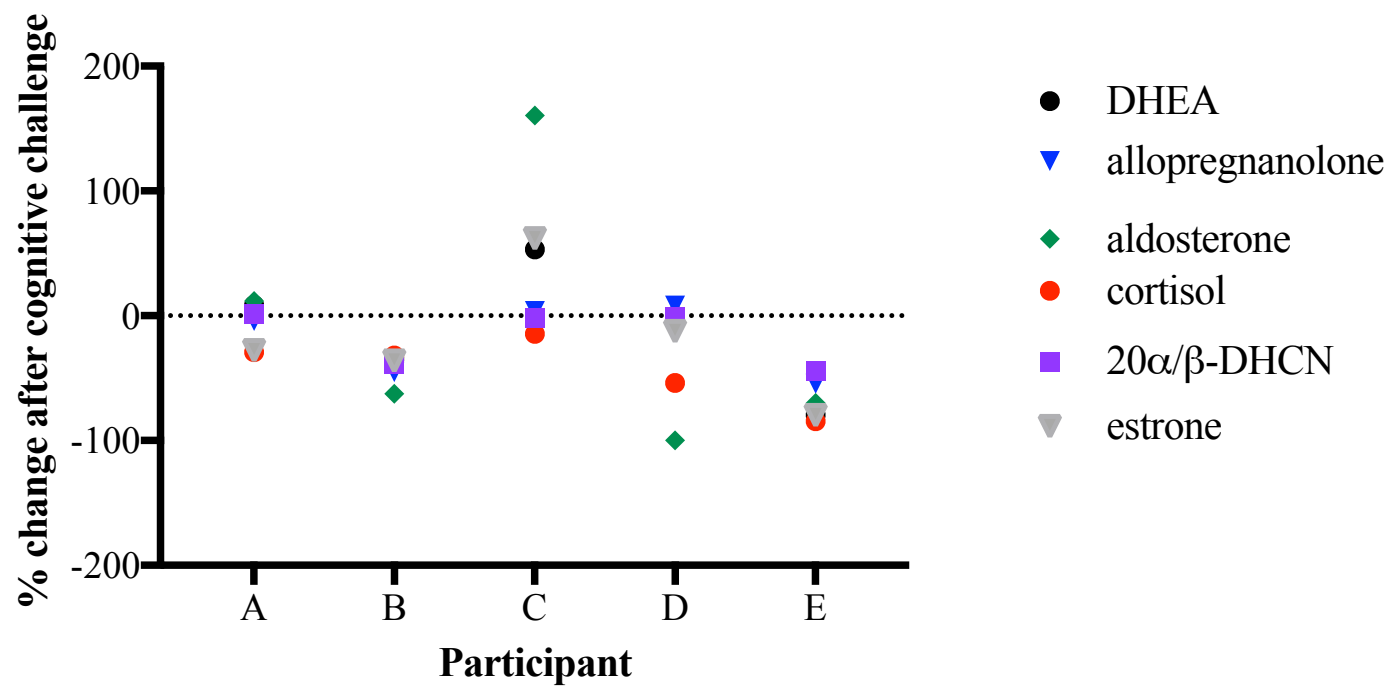

178

179

180

Figure 2. Percent change in palmar sweat steroid biomarker signal after cognitive challenge. 


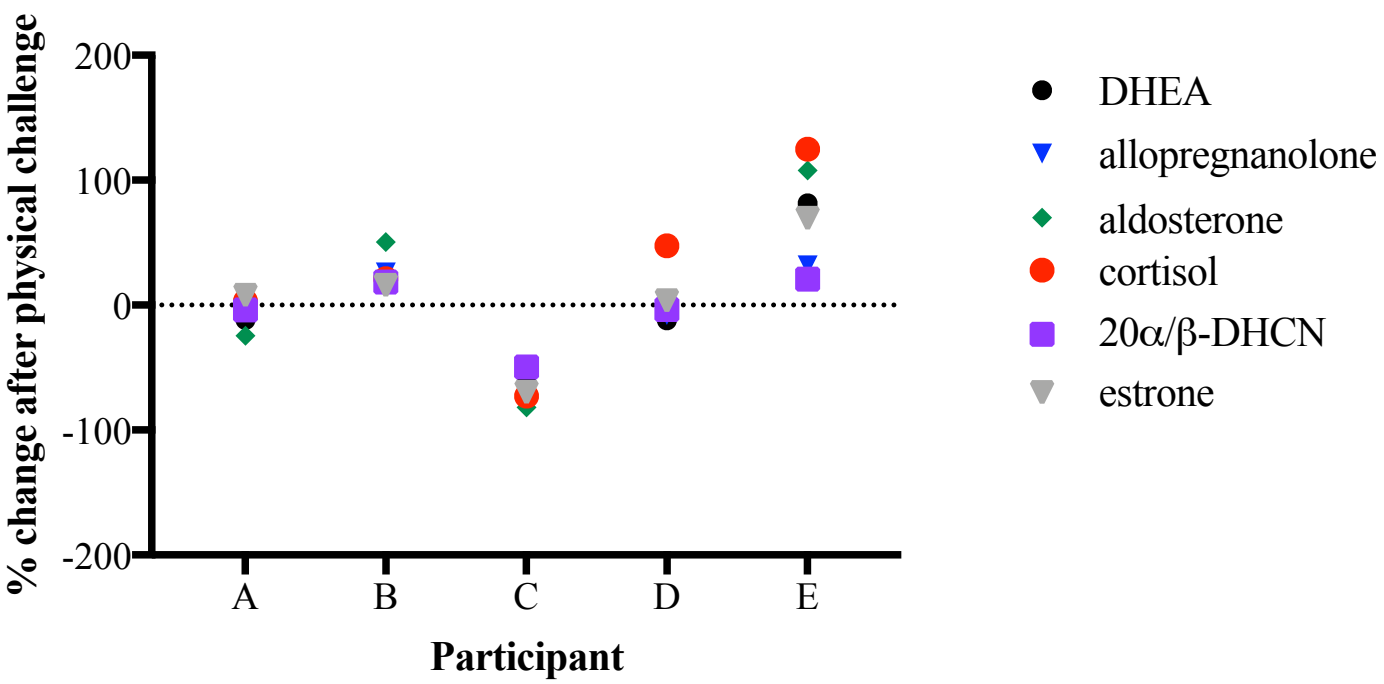

181

182

183

184

185

186

187

188

189

190

191

192

193 for each molecule which may be, in part, influenced by the differential solubility of each steroid

194 in the 70/30 water/ACN extraction solvent and the differential ionization potential for each 195 molecule in the LCMS instrument.

Figure 3. Percent change in palmar sweat steroid biomarker signal after physical challenge.

\subsection{LCMS-ESI-SIM reproducibility}

Eight different steroid biomarkers were used to determine the reproducibility and robustness of the novel LCMS-ESI-SIM method to measure the target steroid biomarkers. The overall relative standard deviation of the LCMS-ESI-SIM measurement, inclusive of sample collection, sample preparation, and instrument variability, was 7\%. Figure 4 shows the linear response of these eight steroids when palmar sweat was extracted using the same method but with different numbers of glass beads. The correlation of each least-squares-fit $\left(\mathrm{R}^{2}\right)$ ranged from $0.977-0.997$ providing confidence in the extraction method. The slope of the lines ranged from 49468 355476 (SIM counts/4mm glass bead) highlighting the different sensitivity of LCMS detection 


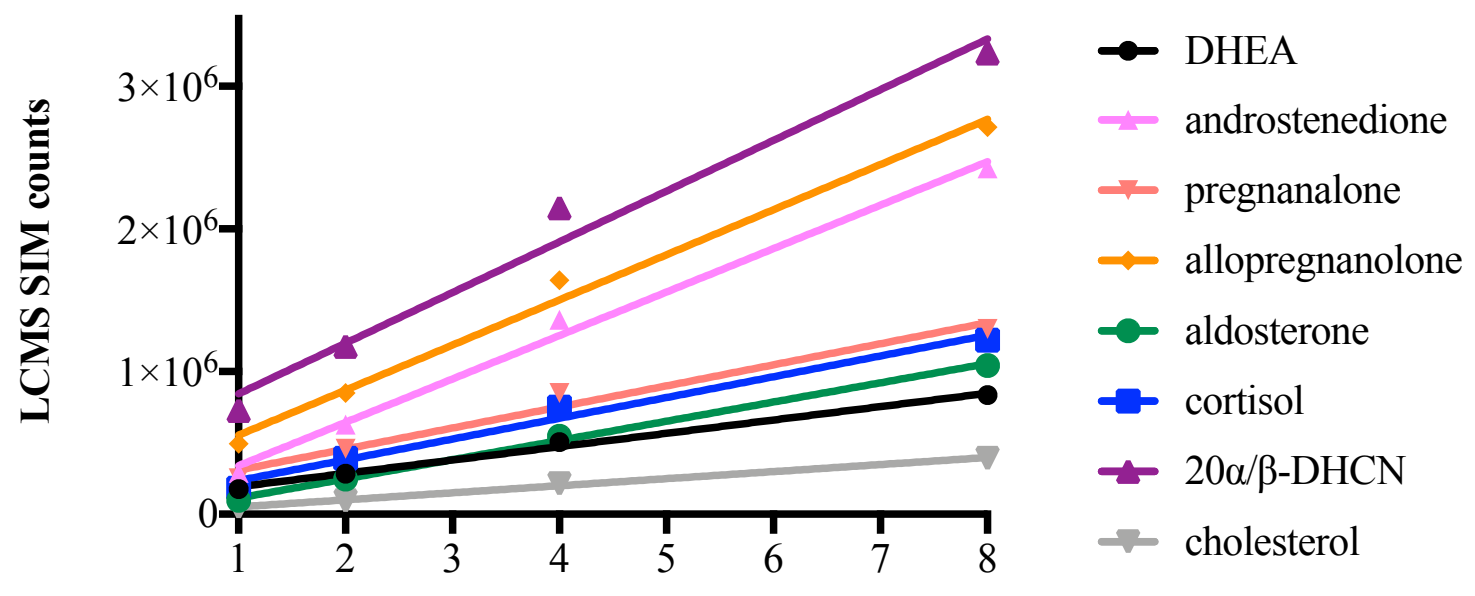

212 Given multiple factors that may influence sweat production, contents, volume, and activation of 213 individual pores (i.e., hydration, health status, medications, diet, gender, topical treatments, etc.),

Figure 4. LCMS-ESI-SIM linear response to increasing glass beads used for extraction of target steroid biomarkers from palmar sweat.

\subsection{Discussion}

In this study, we hypothesized that collection of palmar sweat would yield sweat samples rich in molecular information and provide a sensitive method for measuring biomarker changes on a time scale of minutes in response to stress challenges. The data presented supports this hypothesis and shows that the steroid response is both individualistic and dynamic in real time.

The change of measured biomarkers in response to short cognitive or physical challenges is significant and occurs within the time frame of minutes which agrees with the known time frame response of salivary and sweat cortisol (12). The presentation of the data in Figures $2 \& 3$ as a percent change in biomarker levels allows for comparison between individuals despite the strong individual variability of raw data observed between study participants. 
214 the concentrations of steroid biomarkers in palmar sweat can vary from person to person,

215 sometimes by orders of magnitude. For example, the LCMS-ESI-SIM response for DHEA

216 spanned from $10^{5}$ to $10^{7}$ counts for the participants. The general observed trend in the data was

217 an increase in biomarker concentrations after completion of the physical task, with the exception

218 of a single participant (Participant C) as seen in Figure 3. By comparison, the general trend

219 observed was a decrease in biomarker concentration after completion of the cognitive task as

220 shown in Figure 2. Hyde and colleagues (29) (data not shown) also demonstrated a 4-fold

221 increase in the number of sweat pores activated after completion of the finger tapping physical

222 challenge compared with the N-back cognitive task using thermographic imaging, suggesting

223 that an increase in sweat pore activation contributes in part to the increased biomarker signal

224 measured by LCMS-ESI-SIM. Further studies are needed to deconvolute the individual

225 contributions of increased sweat volume and increased biomarker concentration to the measured

226 LCMS signal for each biomarker.

\section{$228 \quad 5.0$ Conclusion}

229 Non-invasive methods for detecting biomarkers are needed in order to enhance the ability to 230 monitor and track human performance, health, and disease process. The selected biomarkers

231 presented are of particular interest for tracking stress responsiveness and changes in aging

232 populations. U.S. military veterans from Operation Iraqi Freedom (OIF), Operation Enduring

233 Freedom (OEF) and Operation New Dawn (OND) are a timely example of a population with

234 multiple acquired physical and psychiatric conditions where biomarker tracking may be

235 beneficial. OIF/OEF/OND servicemembers and veterans have high rates of PTSD at 23\% (30)

236 and high rates of obesity (BMI $>30 \mathrm{~kg} / \mathrm{m}^{2}$ ) at $44 \%$ (31). While still relatively young, these 
237 complex conditions are unlikely to resolve as this cohort continues to age, further complicating

238 typical age-related physical issues and normal age-related cognitive decline. As previously

239 noted, biomarkers like cortisol, DHEA, allopregnanolone, estrone and aldosterone are all

240 implicated and play a role in the moderation of these conditions. Further, monitoring the status of

241 steroids and immune biomarkers of servicemembers during their active-duty service may provide

242 useable and actionable information necessary to enhance overall performance and potentially

243 decrease the likelihood of negative outcomes after service. In veterans and the general

244 population, biomarker monitoring may help to predict and determine the onset of disease, the

245 course of disease processes, and may influence precision medicine strategies.

247 We have now shown that using non-invasive methods of eccrine sweat collection in very low 248 volumes of sweat is possible via surface capture using glass beads. Multiple biomarkers can be

249 found and measured and may hold promise in monitoring human performance and health. Future 250 studies should focus on applying this methodology to large and diverse cohorts with broad 251 demographics across age ranges, ethnicities, gender, etc., and validating the results against 252 established values in other biofluids such as saliva, serum or urine.

\section{$254 \quad 6.0$ Acknowledgements}

255 The authors would like to thank UArizona Research Innovation and Impact and NIH Grant 256 \#NIHT35 HL07479 (Short-Term Institutional Research Training Grant), the Andrew Weil 257 Center for Integrative Medicine for administrative support, and the Arizona Arthritis Center for 258 facilities support. 


\subsection{References}

261 1. Hu Y, Converse C, Lyons MC, Hsu WH. Neural control of sweat secretion: a review. Br J Dermatol. 2018;178(6):1246-56.

263 2. Baker LB. Physiology of sweat gland function: The roles of sweating and

4. Jia M, Chew W, Feinstein Y, Skeath P, Sternberg E. Quantification of

3. Jia M, Belyavskaya E, Deuster P, Sternberg EM. Development of a sensitive sweat composition in human health. Temperature [Internet]. $2019 \mathrm{Jul}$ 3;6(3):211-59. Available from:

https://doi.org/10.1080/23328940.2019.1632145 microarray immunoassay for the quantitative analysis of neuropeptide $y$. Anal Chem. 2012;84(15):6508-14. Cortisol in Human Eccrine Sweat by Liquid Chromatography - Tandem Mass Spectrometry. Analyst [Internet]. 2016; Available from: http://pubs.rsc.org/en/Content/ArticleLanding/2016/AN/C5AN02387D

5. Sonner Z, Wilder E, Heikenfeld J, Kasting G, Beyette F, Swaile D, et al. The microfluidics of the eccrine sweat gland, including biomarker partitioning,

277 6. Bouslimani A, Porto C, Rath CM, Wang M, Guo Y, Gonzalez A, et al.

278 Molecular cartography of the human skin surface in 3D. Proc Natl Acad Sci. 2015;112(17):E2120-E2129. 
2807 Taylor NAS, Machado-Moreira CA. Regional variations in transepidermal 281 water loss, eccrine sweat gland density, sweat secretion rates and electrolyte 282 composition in resting and exercising humans. Extrem Physiol Med. $283 \quad 2013 ; 2(1): 4$.

284 8. Marques-Deak A, Cizza G, Eskandari F, Torvik S, Christie IC, Sternberg 285 EM, et al. Measurement of cytokines in sweat patches and plasma in healthy women: Validation in a controlled study. J Immunol Methods. 2006;315(1-

9. Cizza G, Marques AH, Eskandari F, Christie IC, Torvik S, Silverman MN, et

al. Elevated Neuroimmune Biomarkers in Sweat Patches and Plasma of

10. Marques AH, Silverman MN, Sternberg EM. Evaluation of stress systems by applying noninvasive methodologies: Measurements of neuroimmune biomarkers in the sweat, heart rate variability and salivary cortisol. Neuroimmunomodulation. 2010;17(3):205-8.

296 11. de Kock N, Acharya SR, Ubhayasekera SJKA, Bergquist J. A Novel Targeted Analysis of Peripheral Steroids by Ultra-Performance Supercritical Fluid Chromatography Hyphenated to Tandem Mass Spectrometry. Sci Rep [Internet]. 2018;8(1):16993. Available from: https://doi.org/10.1038/s41598- 
301 12. Runyon JR, Jia M, Goldstein MR, Skeath P, Abrell L, Chorover J, et al. Dynamic behavior of cortisol and cortisol metabolites in human eccrine sweat. Int J Progn Heal Manag. 2019;

13. Lee DY, Kim E, Choi MH. Technical and clinical aspects of cortisol as a biochemical marker of chronic stress. BMB Rep [Internet]. 2015 Apr 30;48(4):209-16. Available from:

14. Prall SP, Muehlenbein MP. DHEA Modulates Immune Function: A Review http://koreascience.or.kr/journal/view.jsp?kj=E1MBB7\&py=2015\&vnc=v48 $\mathrm{n} 4 \& \mathrm{sp}=209$ of Evidence. Vitam Horm [Internet]. 2018;108:125-44. Available from:

15. Kamin HS, Kertes DA. Cortisol and DHEA in development and psychopathology. Horm Behav [Internet]. 2017 Mar;89:69-85. Available from: https://linkinghub.elsevier.com/retrieve/pii/S0018506X1630215X

315 16. van Zuiden M, Haverkort SQ, Tan Z, Daams J, Lok A, Olff M. DHEA and DHEA-S levels in posttraumatic stress disorder: A meta-analytic review. https://linkinghub.elsevier.com/retrieve/pii/S0083672918300347 
et al. Decreased Cerebrospinal Fluid Allopregnanolone Levels in Women with Posttraumatic Stress Disorder. Biol Psychiatry [Internet]. 2006;60(7):704-13. Available from: http://www.sciencedirect.com/science/article/pii/S0006322306004021

20. Hernandez GD, Brinton RD. Allopregnanolone as a Therapeutic to

Regenerate the Degenerated Brain. In: Brinton RD, Genazzani AR,

18. Paul SM, Pinna G, Guidotti A. Allopregnanolone: From molecular pathophysiology to therapeutics. A historical perspective. Neurobiol Stress [Internet]. 2020 May;12:100215. Available from: https://linkinghub.elsevier.com/retrieve/pii/S2352289520300059

19. Cruz DA, Glantz LA, McGaughey KD, Parke G, Shampine LJ, Kilts JD, et al. Neurosteroid Levels in the Orbital Frontal Cortex of Subjects With PTSD and Controls: A Preliminary Report. Chronic Stress [Internet]. 2019 Jan 1;3:2470547019838570. Available from: https://doi.org/10.1177/2470547019838570 Simoncini T, Stevenson JC, editors. Sex Steroids' Effects on Brain, Heart and Vessels: Volume 6: Frontiers in Gynecological Endocrinology [Internet]. Cham: Springer International Publishing; 2019. p. 111-23. Available from: https://doi.org/10.1007/978-3-030-11355-1_7

21. Mezzullo M, Di Dalmazi G, Fazzini A, Baccini M, Repaci A, Gambineri A, 
et al. Impact of age, body weight and metabolic risk factors on steroid reference intervals in men. Eur J Endocrinol [Internet]. 2020 May;182(5):459-71. Available from:

22. Jasuja GK, Travison TG, Davda M, Rose AJ, Zhang A, Kushnir MM, et al.

23. Ingraham NE, Barakat AG, Reilkoff R, Bezdicek T, Schacker T, Chipman

http://care.diabetesjournals.org/lookup/doi/10.2337/dc12-2477 Sep;36(9):2591-6. Available from: Circulating Estrone Levels Are Associated Prospectively With Diabetes Risk in Men of the Framingham Heart Study. Diabetes Care [Internet]. 2013 
al. Accessing analytes in biofluids for peripheral biochemical monitoring.

Nat Biotechnol [Internet]. 2019; Available from:

362

https://doi.org/10.1038/s41587-019-0040-3

26. Hussain JN, Mantri N, Cohen MM. Working Up a Good Sweat - The

Challenges of Standardising Sweat Collection for Metabolomics Analysis.

Clin Biochem Rev [Internet]. 2017 Feb;38(1):13-34. Available from:

https://pubmed.ncbi.nlm.nih.gov/28798503

27. Kutyshenko VP, Molchanov M, Beskaravayny P, Uversky VN, Timchenko

MA. Analyzing and Mapping Sweat Metabolomics by High-Resolution

NMR Spectroscopy. PLoS One [Internet]. 2011 Dec 14;6(12):e28824.

Available from: https://doi.org/10.1371/journal.pone.0028824

28. Zhou Z, Zare RN. Personal Information from Latent Fingerprints Using Desorption Electrospray Ionization Mass Spectrometry and Machine Learning. Anal Chem [Internet]. 2017 Jan 17;89(2):1369-72. Available from: https://doi.org/10.1021/acs.analchem.6b04498

29. Hyde JN, Majdi MS, Kromenacker B, Wilson RC, Staroschack C, Rodriguez JJ, et al. Thermographic imaging for detection of changes in autonomic nervous system activity and response to varying stress levels through changes 
prevalence of posttraumatic stress disorder in Operation Enduring Freedom/Operation Iraqi Freedom (OEF/OIF) Veterans: a meta-analysis. J Anxiety Disord. 2015 Apr;31:98-107.

383 31. Wischik DL, Magny-Normilus C, Whittemore R. Risk Factors of Obesity in Veterans of Recent Conflicts: Need for Diabetes Prevention. Curr Diab Rep [Internet]. 2019 Sep;19(9):70. Available from: http://ink.springer.com/10.1007/s11892-019-1191-9 